\title{
SYMMETRIC POWERS DO NOT STABILIZE
}

\author{
DANIEL LITT
}

\author{
(Communicated by Lev Borisov)
}

\begin{abstract}
We discuss the stabilization of symmetric products $\operatorname{Sym}^{n}(X)$ of a smooth projective variety $X$ in the Grothendieck ring of varieties. For smooth projective surfaces $X$ with non-zero $h^{0}\left(X, \omega_{X}\right)$, these products do not stabilize; we conditionally show that they do not stabilize in another related sense, in response to a question of $\mathrm{R}$. Vakil and $\mathrm{M}$. Wood. There are analogies between such stabilization, the Dold-Thom theorem, and the analytic class number formula. Finally, we discuss conjectural Hodge-theoretic obstructions to the stabilization of symmetric products. We provide evidence for these obstructions by showing that the Newton polygon of the motivic zeta function associated to a curve equals the Hodge polygon of the curve.
\end{abstract}

\section{INTRODUCTION}

Let $k$ be a field, and let $K_{0}\left(\operatorname{Var}_{k}\right)$ be the Grothendieck ring of varieties over $k$. This is the free abelian group on isomorphism classes $[X]$ of separated, finite type $k$-schemes (varieties), subject to the following relation:

$$
[X]=[Y]+[X \backslash Y] \text { for } Y \hookrightarrow X \text { a closed embedding. }
$$

Multiplication is given by $[X] \cdot[Y]=[X \times Y]$. This ring was introduced by Grothendieck in 1964 [18, (letter of August 16, 1964)] in a letter to Serre. Let $\mathbb{L}:=\left[\mathbb{A}_{k}^{1}\right]$ be the class of the affine line in this ring.

Ravi Vakil and Melanie Wood have conjectured [20, Conjecture 1.25] that in a certain completion of $K_{0}\left(\operatorname{Var}_{k}\right)\left[\mathbb{L}^{-1}\right]$, denoted $\hat{K}$ (defined in Section 2), the limit

$$
\lim _{n \rightarrow \infty} \frac{\left[\operatorname{Sym}^{n} X\right]}{\mathbb{L}^{n \operatorname{dim}(X)}}
$$

exists for connected $X$. They call the existence of this limit "motivic stabilization of symmetric powers" or MSSP; they show this conjecture is true in many cases. The main goal of this paper is to provide some evidence that it is false in general.

We introduce analogous but more accessible claims (False Claims 4 and 5) which hold true in every case where MSSP is known to hold. Furthermore, we show that both false claims are untrue for $k=\mathbb{C}$-counterexamples include smooth projective surfaces $X$ with geometric genus $p_{g}(X) \neq 0$ (Corollary 21) 1 We show that these counterexamples are also counterexamples to MSSP, conditional on the

Received by the editors October 18, 2012 and, in revised form, January 30, 2013.

2010 Mathematics Subject Classification. Primary 14C15, 14C25, 14 G10.

The author was supported by the NSF GRFP.

${ }^{1}$ While this paper was in preparation, Melanie Wood provided several other counterexamples to False Claim 4 22. In particular, her arguments combined with those here show that for smooth projective surfaces with a non-vanishing even plurigenus, False Claim 4 fails. In addition to the results here, this covers e.g. Enriques surfaces and certain surfaces of general type but with $p_{g}=0$. 
truth of either of two well-known conjectures about $K_{0}\left(\operatorname{Var}_{k}\right)$ (the "Cut-and-Paste" conjecture of Larsen and Lunts [1] or the conjecture that $\mathbb{L}$ is not a zero divisor), and thus MSSP is false if either of these conjectures is true (Corollary 23).

Finally, we propose Hodge-theoretic heuristics explaining the (conditional) failure of MSSP for surfaces, and give precise versions (and proofs) of these heuristics for curves. In particular, we prove a motivic analogue of the classical theorem that "the Newton polygon lies above the Hodge polygon" for Weil zeta functions associated to varieties. Namely, if $X$ is a smooth projective curve with a rational point, we show that over a general field, a "motivic Newton polygon" associated to $X$ lies above the Hodge polygon of $X$; this implies the result for Weil zeta functions if $X$ is defined over a finite field (Lemma 31). Over algebraically closed fields of characteristic zero, we show that the Newton and Hodge polygons of curves are equal (Corollary 33). These last results are of independent interest and are contained in Section 5, which can be read independently of Sections 3 and 4.

\section{False Claims and motivation}

Let us first give the statement of MSSP. We define a filtration on $K_{0}\left(\operatorname{Var}_{k}\right)\left[\mathbb{L}^{-1}\right]$, given by dimension. Let $F^{i} K_{0}\left(\operatorname{Var}_{k}\right)\left[\mathbb{L}^{-1}\right] \subset K_{0}\left(\operatorname{Var}_{k}\right)\left[\mathbb{L}^{-1}\right]$ be the (additive) subgroup generated by elements of the form $[X] / \mathbb{L}^{r}$, where $X$ is of dimension $\leq i+r$.

We define the ring $\hat{K}$ to be the completion of $K_{0}\left(\operatorname{Var}_{k}\right)$ at this filtration, that is,

$$
\hat{K}:=\lim _{\longleftarrow} K_{0}\left(\operatorname{Var}_{k}\right)\left[\mathbb{L}^{-1}\right] / F^{i} K_{0}\left(\operatorname{Var}_{k}\right)\left[\mathbb{L}^{-1}\right] .
$$

The ring $\hat{K}$ was initially defined by Kontsevich 99 as the ring in which the values of motivic integrals lie. The MSSP Conjecture takes place in $\hat{K}$ :

Conjecture 1 (Vakil, Wood; motivic stabilization of symmetric powers, or MSSP [20, Conjecture 1.25]). Let $X$ be a connected variety over $k$. Then

$$
\lim _{n \rightarrow \infty} \frac{\left[\operatorname{Sym}^{n} X\right]}{\mathbb{L}^{n \operatorname{dim}(X)}}
$$

exists in $\hat{K}$.

Remark 2. Note that resolution of singularities implies that $F^{i} K_{0}\left(\operatorname{Var}_{k}\right)$ is (additively) generated by elements of the form $[X] / \mathbb{L}^{r}$ with $X$ smooth and proper and of pure dimension $\leq i+r$. In arbitrary characteristic, $F^{i} K_{0}\left(\operatorname{Var}_{k}\right)$ is generated by elements of the form $[X] / \mathbb{L}^{r}$ with $X$ proper and of pure dimension $\leq i+r$. To see this, note that by Noetherian induction, $K_{0}\left(\operatorname{Var}_{k}\right)$ is generated by the classes of affine varieties. But affine varieties are in the subring generated by proper varieties, as they may be compactified, e.g. by considering their scheme-theoretic image under some quasi-projective embedding.

Unfortunately, it is difficult to understand the behavior of $\hat{K}$, as it is unknown whether or not $\mathbb{L}$ is a zero divisor - and even assuming that $\mathbb{L}$ is not a zero divisor, it is not clear that the topology induced by the dimension filtration is separated. Thus, for much of this note, we will instead consider the completion $R$ of $K_{0}\left(\operatorname{Var}_{k}\right)$ at the ideal $(\mathbb{L})$. That is, $R:=\lim _{\longleftarrow} K_{0}\left(\operatorname{Var}_{k}\right) / \mathbb{L}^{n}$.

Remark 3. It is not known whether $\bigcap_{n}\left(\mathbb{L}^{n}\right)=(0)$. That is, it is unclear whether the $\mathbb{L}$-adic topology on $K_{0}\left(\operatorname{Var}_{k}\right)$ is separated. 
Despite the previous remark, the completion $R$ has three advantages:

- Many well-known homomorphisms from $K_{0}\left(\operatorname{Var}_{k}\right)$ to other rings (so-called "motivic measures" [11]) extend continuously to $R$, but not to $\hat{K}$. For example, if $k=\mathbb{F}_{q}$, with $q=p^{n}$, the homomorphism

$$
\psi_{q}: K_{0}\left(\operatorname{Var}_{k}\right) \rightarrow \mathbb{Z}
$$

defined by $\psi_{q}:[X] \mapsto \# X\left(\mathbb{F}_{q}\right)$ extends to a continuous homomorphism $R \rightarrow \mathbb{Z}_{p}$.

- $R$ is easier to work with than $\hat{K}$. In particular, Theorem 12 below (due to Larsen and Lunts, [11, Proposition 2.7]) shows that $K_{0}\left(\operatorname{Var}_{k}\right) / \mathbb{L}$ remembers exactly the stable birational geometry of smooth projective varieties, so convergence of limits in $R$ "to first order" has a geometric meaning.

- If $k$ is algebraically closed of characteristic zero, we may define a surjection of topological rings

$$
\mathbb{D}: R\left[\mathbb{L}^{-1}\right] \rightarrow \hat{K}
$$

(see Remark 18); if $\mathbb{L}$ is not a zero-divisor, then $\mathbb{D}$ is an isomorphism. For a smooth projective curve $X$,

$$
\mathbb{D}\left(\left[\operatorname{Sym}^{n}(X)\right]\right)=\frac{\left[\operatorname{Sym}^{n}(X)\right]}{\mathbb{L}^{n}} .
$$

For a smooth projective surface $S$,

$$
\mathbb{D}\left(\left[\operatorname{Hilb}^{n}(S)\right]\right)=\frac{\left[\operatorname{Sym}^{n}(S)\right]}{\mathbb{L}^{2 n}} \bmod F^{-1} K_{0}\left(\operatorname{Var}_{k}\right)\left[\mathbb{L}^{-1}\right] .
$$

Thus, we consider the following False Claim as an alternative to Conjecture 1. Much of the work in this paper will be aimed at examining the circumstances in which this claim fails.

False Claim 4. For connected $X$, the limit

$$
\lim _{n \rightarrow \infty}\left[\operatorname{Sym}^{n} X\right]
$$

exists in $R$.

False Claim 4 would immediately imply:

False Claim 5. For $X$ connected and $n, m \gg 0,\left[\operatorname{Sym}^{n} X\right]=\left[\operatorname{Sym}^{m} X\right]$ in $K_{0}\left(\operatorname{Var}_{k}\right) / \mathbb{L}$.

But we will disprove False Claim 5 in Section 4, for $k$ an algebraically closed field of characteristic 0 .

Before disproving these claims, however, we would like to take the odd step of motivating our False Claims 4 and 5, as well as the MSSP conjecture of Vakil and Wood. In particular, we will make a case that these false claims are natural, despite their falsehood. We will see (Corollary 23) that if False Claim 5 fails for a smooth projective surface $S$ over an algebraically closed field of characteristic zero, MSSP fails as well, conditional on either of Conjectures 13 or 14 .

First, let $C$ be a smooth proper genus $g$ curve over a field $k$, with a $k$-rational point. Then for $n \gg 0, \operatorname{Sym}^{n} C$ is a (Zariski) $\mathbb{P}^{n-g}$-bundle over $\operatorname{Jac}(C)$, and so

$$
\left[\operatorname{Sym}^{n} C\right]=\left(1+\mathbb{L}+\cdots+\mathbb{L}^{n-g}\right)[\operatorname{Jac}(C)]
$$

in $K_{0}\left(\operatorname{Var}_{k}\right)$. Thus the limit $\lim _{n \rightarrow \infty}\left[\operatorname{Sym}^{\mathrm{n}} C\right]$ clearly exists in $R$ and equals

$$
[\operatorname{Jac}(C)]\left(1+\mathbb{L}+\mathbb{L}^{2}+\cdots\right) .
$$


So False Claim 4 holds for smooth proper curves with a rational point.

Similarly, False Claim 4 holds for connected rational or uniruled surfaces and for connected varieties $X$ whose classes $[X] \in K_{0}\left(\operatorname{Var}_{k}\right)$ are polynomial in $\mathbb{L}$, e.g. (split) affine algebraic groups and their homogeneous spaces. The proofs of these claims are not difficult, so we omit them. These examples also provide evidence for MSSP; every $X$ for which MSSP is known to hold also satisfies False Claim 4.

MSSP and False Claim 4, when true, provide an algebro-geometric analogue of the following beautiful theorem of Dold and Thom.

Theorem 6 (Dold-Thom [3]). Let $X$ be a connected CW complex with basepoint $x_{0}$. Let $S P^{\infty}(X)$ be the direct limit of the spaces $\operatorname{Sym}^{n} X$, under the maps $\operatorname{Sym}^{n} X \hookrightarrow \operatorname{Sym}^{n+1} X$ given by $\left(x_{1}, x_{2}, \cdots, x_{n}\right) \mapsto\left(x_{0}, x_{1}, \ldots, x_{n}\right)$. Then there is an identification

$$
S P^{\infty}(X) \simeq_{w} \prod_{i>0} K\left(H_{i}(X, \mathbb{Z}), i\right)
$$

where $K(G, n)$ is an Eilenberg-MacLane space; namely, a space with $\pi_{n}(K(G, n))=$ $G, \pi_{m}(K(G, n))=\{0\}$ for $m \neq n$. The symbol $\simeq_{w}$ denotes weak equivalence.

Compare this to the situation in False Claim 4 for $X$ a smooth proper curve of genus $g$ over $\mathbb{C}$. In this case, $\operatorname{Jac}(X)$ has the homotopy type of $K\left(H_{1}(X, \mathbb{Z}), 1\right)$ by construction, and $H_{2}(X, \mathbb{Z})=\mathbb{Z}$, so $K\left(H_{2}(X, \mathbb{Z}), 2\right) \simeq_{w} \mathbb{C P}^{\infty} \simeq \lim _{\mathbb{C P}}{ }^{n}$, which we may view as being represented by the class

$$
\left[\mathbb{C P}^{\infty}\right]:=\lim _{n \rightarrow \infty}\left[\mathbb{P}^{n}\right]=1+\mathbb{L}+\mathbb{L}^{2}+\cdots
$$

in $R$. Thus the Dold-Thom theorem gives

$$
\lim _{\longrightarrow} \operatorname{Sym}^{n} X \simeq_{w} K\left(\mathbb{Z}^{2 g}, 1\right) \times K(\mathbb{Z}, 2) \simeq_{w} \operatorname{Jac}(X) \times \mathbb{C P}^{\infty} .
$$

Likewise, our computation (11) above gives

$$
\lim _{n \rightarrow \infty}\left[\operatorname{Sym}^{n} X\right]=[\operatorname{Jac}(C)]\left[\mathbb{C P}^{\infty}\right]
$$

in $R$. So we may view the Dold-Thom theorem as evidence for False Claim 4 ,

Finally, consider the case $k=\mathbb{F}_{q}$, where $q=p^{n}$. In this case, there is a pointcounting ring homomorphism $\psi_{q}: K_{0}\left(\operatorname{Var}_{k}\right) \rightarrow \mathbb{Z}$ sending a variety $X$ to $\# X\left(\mathbb{F}_{q}\right)$. By analogy to the zeta function

$$
\zeta_{X}(t):=\exp \left(\sum_{n} \frac{\# X\left(\mathbb{F}_{q^{n}}\right)}{n} t^{n}\right)
$$

appearing in the Weil conjectures, Kapranov [8, 1.3] introduced the following motivic zeta function associated to a variety $X$ over an arbitrary field:

$$
Z_{X}^{m o t}(t)=\sum_{i=0}^{\infty}\left[\operatorname{Sym}^{n} X\right] t^{n} \in K_{0}\left(\operatorname{Var}_{k}\right)[[t]] .
$$

In the case that $k$ is finite,

$$
\psi_{q}\left(Z_{X}^{m o t}(t)\right)=\zeta_{X}(t)
$$

where $\psi_{q}$ denotes the homomorphism $K_{0}\left(\operatorname{Var}_{k}\right)[[t]] \rightarrow \mathbb{Z}[[t]]$ induced by applying the point-counting map coefficientwise.

We have

$$
\lim _{n \rightarrow \infty}\left[\operatorname{Sym}^{n} X\right]=\left.(1-t) Z_{X}^{m o t}(t)\right|_{t=1}
$$


formally, where both expressions are evaluated in $R$. If $X$ is a curve or a rational or uniruled surface, $(1-t) Z_{X}^{m o t}(t)$ is everywhere convergent as a power series over $R$. If $X$ is a smooth proper curve, the limit on the left specializes under $\psi_{q}$ to the "analytic class number formula" for the zeta functions appearing in the Weil conjectures. We have that

$$
\operatorname{res}_{t=1} \zeta_{X}(t)=\frac{\# \operatorname{Jac}(X)\left(\mathbb{F}_{q}\right)}{1-q}
$$

and likewise

$$
\left.(1-t) Z_{X}^{m o t}(t)\right|_{t=1}=\frac{[\operatorname{Jac}(X)]}{1-\mathbb{L}} .
$$

Thus we may view False Claim 4 or MSSP as analogues of analytic class number formulas. To put it another way, these claims are analogues of the fact that the zeta functions appearing in the Weil conjectures have a pole of order 1 at $t=1$ assuming the power series expansion for $(1-t) Z_{X}^{m o t}(t)$ is valid at $t=1$. We will expand on this last heuristic in Section 5.

Remark 7. We refer to equations (2) and (3) as "analytic class number formulas" because of their resemblance to the analytic class number formula for Dedekind zeta functions associated to number fields.

Remark 8. By analogy to the Weil conjectures, one might guess that $Z_{X}^{m o t}(t)$ is the power series associated to a rational function with coefficients in $K_{0}\left(\operatorname{Var}_{k}\right)$. Kapranov shows that this is true for curves with a rational point [8, (1.3.5)(a)], where the hypothesis of the existence of a rational point is left implicit. For curves with no rational point the argument does not work. The issue is that the usual Picard functor is not representable in this case, and so $\operatorname{Sym}^{n}(X)$ is not a projective space bundle over $\operatorname{Pic}^{n}(X)$, which is an obstruction to Kapranov's argument. It is unclear to the author if this issue can be rectified. On the other hand, Larsen and Lunts have shown [11,12 that these zeta functions are not rational over $K_{0}\left(\operatorname{Var}_{k}\right)$ for most surfaces. The question of the rationality of $Z_{X}^{m o t}(t)$ over $K_{0}\left(\operatorname{Var}_{k}\right)\left[\mathbb{L}^{-1}\right]$ is open.

The plan for the rest of this paper is as follows. In Section 3 we will introduce several facts and conjectures about $K_{0}\left(\operatorname{Var}_{k}\right)$ and discuss their interplay. In Section 4 we will disprove False Claims 4 and 5 and deduce the conditional falsity of MSSP in the case of smooth projective surfaces $X$ with non-vanishing $H^{0}\left(X, \omega_{X}\right)$. In Section 5 we will propose Hodge-theoretic heuristics for the failure of MSSP and give evidence for them in terms of the "Newton polygons" of Kapranov zeta functions $Z_{X}^{m o t}(t)$ of curves.

\section{PReliminaries AND DisCUSSION}

From here on, unless otherwise stated, $k$ will be algebraically closed of characteristic zero.

In [1], Bittner gives the following useful presentation of $K_{0}\left(\operatorname{Var}_{k}\right)$ - the proof uses resolution of singularities and weak factorization of rational maps. We will use her description of $K_{0}\left(\operatorname{Var}_{k}\right)$ and some of her constructions to relate MSSP to False Claims 4 and 5 . 
Theorem 9 (Bittner [1, Theorem 3.1]). $K_{0}\left(\operatorname{Var}_{k}\right)$, for $k$ algebraically closed and of characteristic zero, is generated by the classes of smooth proper $k$-varieties, subject only to the following relations:

$$
\left[\mathrm{Bl}_{Y}(X)\right]-[E]=[X]-[Y]
$$

for $X$ proper, $Y$ a smooth closed subvariety of $X$, and $E$ the exceptional divisor of the blowup $\mathrm{Bl}_{Y}(X)$.

Bittner uses this presentation to construct a "duality map" $\mathbb{D}: K_{0}\left(\operatorname{Var}_{k}\right) \rightarrow$ $K_{0}\left(\operatorname{Var}_{k}\right)\left[\mathbb{L}^{-1}\right]$, which we will use heavily.

Corollary 10 (Duality map [1, Corollary 3.4]). There exists a unique ring homomorphism $\mathbb{D}: K_{0}\left(\operatorname{Var}_{k}\right) \rightarrow K_{0}\left(\operatorname{Var}_{k}\right)\left[\mathbb{L}^{-1}\right]$ with

$$
\mathbb{D}([X])=\frac{[X]}{\mathbb{L}^{\operatorname{dim}(X)}}
$$

for $X$ smooth and proper.

Note that $\mathbb{D}(\mathbb{L})=\mathbb{D}\left(\mathbb{P}^{1}\right)-\mathbb{D}([\{\mathrm{pt}\}])=\mathbb{L}^{-1}$. Thus $\mathbb{D}$ induces a map

$$
\mathbb{D}^{\prime}: K_{0}\left(\operatorname{Var}_{k}\right)\left[\mathbb{L}^{-1}\right] \rightarrow K_{0}\left(\operatorname{Var}_{k}\right)\left[\mathbb{L}^{-1}\right]
$$

satisfying $\mathbb{D}^{\prime} \circ \mathbb{D}^{\prime}=$ id.

We will need one further result on $K_{0}\left(\operatorname{Var}_{k}\right)$, due to Larsen and Lunts [11; it also follows from Bittner's presentation of $K_{0}\left(\operatorname{Var}_{k}\right)$.

Definition 11 (Stable birationality). Let $X$ and $Y$ be two varieties; recall that $X$ is stably birational to $Y$ if $X \times \mathbb{A}^{n}$ is birational to $Y \times \mathbb{A}^{m}$ for some $m, n$. If $k$ is separably closed, $S B$ denotes the monoid of stable birational equivalence classes of smooth, connected, proper $k$-varieties under the operation of Cartesian product.

Theorem 12 (Larsen and Lunts, [11, Proposition 2.7]). Let $k$ be algebraically closed of characteristic zero. Then there is a ring homomorphism

$$
s b: K_{0}\left(\operatorname{Var}_{k}\right) / \mathbb{L} \rightarrow \mathbb{Z}[S B],
$$

sending the class of a smooth proper variety to its stable birational equivalence class; $s b$ is an isomorphism.

For future reference, we will list here two conjectures about $K_{0}\left(\operatorname{Var}_{k}\right)$; we will show that MSSP fails conditional on the truth of either of these conjectures.

Conjecture 13 (Cut-and-Paste conjecture, Larsen and Lunts 11]). Let $X$ and $Y$ be varieties over $k$. If $[X]=[Y]$ in $K_{0}\left(\operatorname{Var}_{k}\right)$, then there exist disjoint locally closed subvarieties $X_{i}$ of $X, Y_{i}$ of $Y$ such that $X=\bigcup X_{i}, Y=\bigcup Y_{i}$, and $X_{i} \simeq Y_{i}$ for all $i$.

Conjecture 14 (Cancellation of the Lefschetz motive [2, 3.3], [13, remarks after Assertion 1]). $\mathbb{L}$ is not a zero divisor in $K_{0}\left(\operatorname{Var}_{k}\right)$.

This latter conjecture is a standard open question about the Grothendieck ring of varieties, e.g. in [13, Remark 16], [10, 7.1].

We also record consequences of these conjectures which will be required later. 
Proposition 15 (Stable birationality in $\hat{K})$. Let $X$ and $Y$ be irreducible varieties over $k$. Then if the Cut-and-Paste conjecture (Conjecture 13) holds for $k$, we have that

$$
\frac{[X]}{\mathbb{L}^{\operatorname{dim}(X)}}=\frac{[Y]}{\mathbb{L}^{\operatorname{dim}(Y)}}
$$

in $F^{0} K_{0}\left(\operatorname{Var}_{k}\right)\left[\mathbb{L}^{-1}\right] / F^{-1} K_{0}\left(\operatorname{Var}_{k}\right)\left[\mathbb{L}^{-1}\right]$ only if $X$ is stably birational to $Y$.

Proof. The idea of the argument is to translate this equality into an equality in $K_{0}\left(\operatorname{Var}_{k}\right)$, and then apply the Cut-and-Paste conjecture there.

If

$$
\frac{[X]}{\mathbb{L}^{\operatorname{dim}(X)}}=\frac{[Y]}{\mathbb{L}^{\operatorname{dim}(Y)}}
$$

in $F^{0} K_{0}\left(\operatorname{Var}_{k}\right)\left[\mathbb{L}^{-1}\right] / F^{-1} K_{0}\left(\operatorname{Var}_{k}\right)\left[\mathbb{L}^{-1}\right]$, there are equidimensional varieties $S_{i}$ such that

$$
\frac{[X]}{\mathbb{L}^{\operatorname{dim}(X)}}-\frac{[Y]}{\mathbb{L}^{\operatorname{dim}(Y)}}=\sum_{i \in I} a_{i} \frac{\left[S_{i}\right]}{\mathbb{L}^{n_{i}}}
$$

in $K_{0}\left(\operatorname{Var}_{k}\right)\left[\mathbb{L}^{-1}\right]$, where $n_{i}>\operatorname{dim}\left(S_{i}\right), a_{i} \in \mathbb{Z}$, and $I$ is a finite index set. Equivalently, for some $N \gg 0$,

$$
\mathbb{L}^{N-\operatorname{dim}(X)}[X]-\mathbb{L}^{N-\operatorname{dim}(Y)}[Y]=\sum_{i \in I} a_{i} \mathbb{L}^{N-n_{i}}\left[S_{i}\right]
$$

in $K_{0}\left(\operatorname{Var}_{k}\right)$. Rearranging terms, we have for $J \subset I, J:=\left\{i \in I \mid a_{i}<0\right\}$ that

$$
\mathbb{L}^{N-\operatorname{dim}(X)}[X]+\sum_{j \in J}\left|a_{j}\right| \mathbb{L}^{N-n_{j}}\left[S_{j}\right]=\mathbb{L}^{N-\operatorname{dim}(Y)}[Y]+\sum_{i \in I \backslash J}\left|a_{i}\right| \mathbb{L}^{N-n_{i}}\left[S_{i}\right] .
$$

Now, if the Cut-and-Paste conjecture holds, the equality above implies that we may write

$$
X^{\prime}=\left(\mathbb{A}^{N-\operatorname{dim}(X)} \times X\right) \cup \bigcup_{j \in J} \mathbb{A}^{N-n_{j}} \times(\underbrace{S_{j} \cup \cdots \cup S_{j}}_{\left|a_{j}\right| \text { times }})
$$

and

$$
Y^{\prime}=\left(\mathbb{A}^{N-\operatorname{dim}(Y)} \times Y\right) \cup \bigcup_{i \in I \backslash J} \mathbb{A}^{N-n_{i}} \times(\underbrace{S_{i} \cup \cdots \cup S_{i}}_{\left|a_{i}\right| \text { times }})
$$

as disjoint unions of isomorphic locally closed subsets. That is, $X^{\prime}$ and $Y^{\prime}$ are equidecomposable. But $X^{\prime}, Y^{\prime}$ have exactly one connected component of dimension $N$-respectively, $\mathbb{A}^{N-\operatorname{dim}(X)} \times X$ and $\mathbb{A}^{N-\operatorname{dim}(Y)} \times Y$-and all other connected components have dimension less than $N$, as $n_{i}>\operatorname{dim}\left(S_{i}\right)$. So $\mathbb{A}^{N-\operatorname{dim}(X)} \times$ $X, \mathbb{A}^{N-\operatorname{dim}(Y)} \times Y$ must be birational. Thus $X$ and $Y$ are stably birational.

We also have a similar result contingent on the truth of Conjecture 14.

Proposition 16. Suppose $\mathbb{L}$ is not a zero divisor in $K_{0}\left(\operatorname{Var}_{k}\right)$ (Conjecture 14). Then

$$
F^{0} K_{0}\left(\operatorname{Var}_{k}\right)\left[\mathbb{L}^{-1}\right] / F^{-1} K_{0}\left(\operatorname{Var}_{k}\right)\left[\mathbb{L}^{-1}\right] \simeq K_{0}\left(\operatorname{Var}_{k}\right) / \mathbb{L}
$$

Proof. We first define a map

$$
f: K_{0}\left(\operatorname{Var}_{k}\right) / \mathbb{L} \rightarrow F^{0} K_{0}\left(\operatorname{Var}_{k}\right)\left[\mathbb{L}^{-1}\right] / F^{-1} K_{0}\left(\operatorname{Var}_{k}\right)\left[\mathbb{L}^{-1}\right] .
$$

This map is induced by $\mathbb{D}$; we must show that $(\mathbb{L})$ maps into $F^{-1} K_{0}\left(\operatorname{Var}_{k}\right)\left[\mathbb{L}^{-1}\right]$ and that the image of $\mathbb{D}$ is contained in $F^{0} K_{0}\left(\operatorname{Var}_{k}\right)\left[\mathbb{L}^{-1}\right]$. For the former statement, 
note that the ideal $(\mathbb{L})$ is generated by elements of the form $\mathbb{L}[X]$, where $X$ is smooth, connected, and proper. Then

$$
\mathbb{D}(\mathbb{L}[X])=\frac{[X]}{\mathbb{L}^{\operatorname{dim}(X)+1}} \in F^{-1} K_{0}\left(\operatorname{Var}_{k}\right)\left[\mathbb{L}^{-1}\right],
$$

as desired. The latter statement follows analogously (in fact we have that $\mathbb{D}\left(\left(\mathbb{L}^{n}\right)\right)$ equals $F^{-n} K_{0}\left(\operatorname{Var}_{k}\right)\left[\mathbb{L}^{-1}\right]$ for all $\left.n \geq 0\right)$.

If $\mathbb{L}$ is not a zero divisor, we may define an inverse map. By the previous observation, $\mathbb{D}^{\prime}\left(F^{0} K_{0}\left(\operatorname{Var}_{k}\right)\left[\mathbb{L}^{-1}\right]\right)$ is exactly the image of $K_{0}\left(\operatorname{Var}_{k}\right)$ in $K_{0}\left(\operatorname{Var}_{k}\right)\left[\mathbb{L}^{-1}\right]$ via the natural inclusion; as $\mathbb{L}$ is not a zero divisor, this image is isomorphic to $K_{0}\left(\operatorname{Var}_{k}\right)$ itself. So $\mathbb{D}^{\prime}$ induces a map $g: F^{0} K_{0}\left(\operatorname{Var}_{k}\right)\left[\mathbb{L}^{-1}\right] \rightarrow K_{0}\left(\operatorname{Var}_{k}\right)$. We need to check that $F^{-1} K_{0}\left(\operatorname{Var}_{k}\right)$ maps to $(\mathbb{L})$. But the verification proceeds as in the previous paragraph.

Corollary 17. If $\mathbb{L}$ is not a zero divisor in $K_{0}\left(\operatorname{Var}_{k}\right)$,

$$
F^{0} K_{0}\left(\operatorname{Var}_{k}\right)\left[\mathbb{L}^{-1}\right] / F^{-1} K_{0}\left(\operatorname{Var}_{k}\right)\left[\mathbb{L}^{-1}\right] \simeq \mathbb{Z}[S B] .
$$

If $X$ is smooth and proper, this isomorphism sends $[X] / \mathbb{L}^{\operatorname{dim}(X)}$ to the stable birational equivalence class of $X$.

Proof. This follows immediately from Theorem 12 and Proposition 16.

Remark 18 . Note that $\mathbb{D}$ extends to a continuous surjection $\mathbb{D}: R\left[\mathbb{L}^{-1}\right] \rightarrow \hat{K}$. If $\mathbb{L}$ is not a zero divisor, the methods of Proposition [16] show that this is an isomorphism. As claimed in Section 2, for a smooth projective curve $X$,

$$
\mathbb{D}\left(\left[\operatorname{Sym}^{n}(X)\right]\right)=\frac{\left[\operatorname{Sym}^{n}(X)\right]}{\mathbb{L}^{n}} .
$$

For a smooth projective surface $S$,

$$
\mathbb{D}\left(\left[\operatorname{Hilb}^{n}(S)\right]\right)=\frac{\left[\operatorname{Sym}^{n}(S)\right]}{\mathbb{L}^{2 n}} \bmod F^{-1} K_{0}\left(\operatorname{Var}_{k}\right)\left[\mathbb{L}^{-1}\right],
$$

where we use the fact that $\operatorname{Hilb}^{n}(S)$ is smooth and projective for $S$ smooth and projective [5, p. 167].

\section{Stable Birationality of Symmetric POWERS}

The main geometric content of this section is the following:

Theorem 19. Let $X$ be a smooth connected projective surface with $h^{0}\left(X, \omega_{X}\right) \neq 0$. Let $m$ be a non-negative integer. Then for all sufficiently large $n, \operatorname{Sym}^{n}(X)$ is not stably birational to $\operatorname{Sym}^{m}(X)$.

The idea of the proof is to produce a moving family of unirational subvarieties of $\operatorname{Sym}^{n}(X)$ of high dimension, contradicting the following theorem of Mumford.

Theorem 20 (Mumford, 15, corollary on page 203). There exists a codimensionone subvariety $W$ of $\operatorname{Sym}^{n}(X)$ so that if $Y \subset \operatorname{Sym}^{n}(X) \backslash W$ consists entirely of rationally equivalent 0 -cycles, then $Y$ has dimension at most $n$.

Mumford's proof uses the so-called "symplectic argument" [21, Chapter 10]. The idea is to show that a connected subvariety of $\operatorname{Sym}^{n}(X)$ consisting of rationally equivalent zero-cycles and containing a generic 0 -cycle must lie tangent to an isotropic subspace of any two-form on $\operatorname{Sym}^{n}(X)$. One can construct generically 
non-degenerate two-forms on the smooth locus of $\operatorname{Sym}^{n}(X)$ given any non-zero two-form on $X$, giving an upper bound on the dimension of most such varieties.

Proof of Theorem 19, Without loss of generality $n>m$. Assume for the sake of contradiction that $\operatorname{Sym}^{n}(X) \times \mathbb{A}^{l}$ is birational to $\operatorname{Sym}^{m}(X) \times \mathbb{A}^{2 n-2 m+l}$ for some $l$. Then there exists a variety $U$ which may be embedded as a dense open subvariety of both $\operatorname{Sym}^{n}(X) \times \mathbb{A}^{l}$ and $\operatorname{Sym}^{m}(X) \times \mathbb{A}^{2 n-2 m+l}$.

A non-empty fiber of the projection map $\pi_{m}: U \rightarrow \operatorname{Sym}^{m}(X)$ is a dense open subset of $\mathbb{A}^{2 n-2 m+l}$, and is thus of dimension $2 n-2 m+l$. Choosing any $k$-point $x$ in the image of the other projection map $\pi_{n}: U \rightarrow \operatorname{Sym}^{n}(X)$, there exists some $k$-point $y \in \operatorname{Sym}^{m}(X)$ so that $x \in \pi_{n}\left(\pi_{m}^{-1}(y)\right)$.

Choosing $x \in \operatorname{Sym}^{n}(X)$ lying away from the subvariety $W$ of $\operatorname{Sym}^{n}(X)$ coming from Theorem 20. we choose $y$ as above and let $Y=\pi_{n}\left(\pi_{m}^{-1}(y)\right)$. As $\pi_{m}^{-1}(y)$ is an open subset of affine space, $Y$ is unirational; furthermore $Y$ has dimension $2 n-2 m$, as the non-empty fibers of $\pi_{n}$ have dimension $l$. As $Y$ is unirational, points in it correspond to rationally equivalent 0-cycles. So for $n>2 m, Y \backslash(Y \cap W)$ is a subvariety of $\operatorname{Sym}^{n}(X) \backslash W$ consisting of rationally equivalent 0 -cycles, of dimension larger than $n$, which contradicts Theorem 20.

Corollary 21 (False Claims 4 and 5 are false). Let $X$ be as in Theorem 19. Then the classes $\left[\operatorname{Sym}^{n}(X)\right]$ do not stabilize in $K_{0}\left(\operatorname{Var}_{k}\right) / \mathbb{L}$.

Before proving this corollary, we will need the following result of Göttsche.

Theorem 22 (Göttsche, [7, Theorem 1.1]). Let $S$ be a smooth projective surface over $k$. Then in $K_{0}\left(\operatorname{Var}_{k}\right)$ we have

$$
\left[\operatorname{Hilb}^{n}(S)\right]=\sum_{\left(1^{\alpha_{1}}, \ldots, n^{\alpha_{n}}\right) \in P(n)} \mathbb{L}^{n-|\alpha|} \prod_{i=1}^{n}\left[\operatorname{Sym}^{\alpha_{i}}(S)\right] .
$$

Here $\operatorname{Hilb}^{n}(X)$ is the Hilbert scheme of length $n$ subschemes of $X$, and $P(n)$ is the set of partitions of $n$. If $\alpha \in P(n)$ we write $\alpha=\left(1^{\alpha_{1}}, 2^{\alpha_{2}}, \cdots, n^{\alpha_{n}}\right)$ and define $|\alpha|=\sum_{i} \alpha_{i}$.

An immediate consequence is that

$$
\left[\operatorname{Hilb}^{n}(S)\right]=\left[\operatorname{Sym}^{n}(S)\right] \bmod \mathbb{L} .
$$

Proof of Corollary 21. As $\operatorname{Hilb}^{n}(X)$ is birational to $\operatorname{Sym}^{n}(X)$ [5, p. 161] via the Hilbert-Chow morphism, we have from Theorem 19 that $\operatorname{Hilb}^{n}(X)$ is not stably birational to $\operatorname{Hilb}^{m}(X)$ for $n \gg m$. Furthermore, as $X$ is a smooth projective surface, $\operatorname{Hilb}^{n}(X)$ is smooth and projective [5, p. 167], so [Hilb $\left.{ }^{n}(X)\right]$ maps to its stable birational equivalence class via the isomorphism $s b: K_{0}\left(\operatorname{Var}_{k}\right) / \mathbb{L} \rightarrow \mathbb{Z}[S B]$. But the images of the $\operatorname{Hilb}^{n}(X)$ in $\mathbb{Z}[S B]$ do not stablize, so the classes $\left[\operatorname{Hilb}^{n}(X)\right.$ ] in $K_{0}\left(\operatorname{Var}_{k}\right) / \mathbb{L}$ do not stabilize either. Finally,

$$
\left[\operatorname{Hilb}^{n}(X)\right]=\left[\operatorname{Sym}^{n}(X)\right] \bmod \mathbb{L},
$$

so the classes $\left[\operatorname{Sym}^{n}(X)\right]$ do not stabilize, as desired.

Corollary 23 (Conditional falsity of MSSP). If either (a) Conjecture 13 (Cutand-Paste) or (b) Conjecture 14 ( $\mathbb{L}$ is not a zero divisor) holds for $k$, then MSSP fails for $X$ as in Theorem 19 . 
Proof. The fact that (a) Conjecture 13 implies the failure of MSSP follows immediately from Theorem 19 and Proposition [15. For (b), note that

in

$$
\frac{\left[\operatorname{Hilb}^{n}(X)\right]}{\mathbb{L}^{2 n}}=\frac{\left[\operatorname{Sym}^{n}(X)\right]}{\mathbb{L}^{2 n}}
$$

$$
F^{0} K_{0}\left(\operatorname{Var}_{k}\right)\left[\mathbb{L}^{-1}\right] / F^{-1} K_{0}\left(\operatorname{Var}_{k}\right)\left[\mathbb{L}^{-1}\right]
$$

as $\operatorname{Sym}^{n}(X)$ and $\operatorname{Hilb}^{n}(X)$ are birational. But $\operatorname{Hilb}^{n}(X)$ is smooth and proper, so its image in $\mathbb{Z}[S B]$ via the map defined in Proposition [16] and Corollary [17 is its stable birational equivalence class. These images do not stabilize, by Theorem 19 .

Remark 24. We sketch here a proof that False Claims 4 and 5 also fail for $k=\mathbb{F}_{q}$ a finite field; this also falsifies MSSP conditional on resolution of singularities, weak factorization of birational maps, and either of Conjectures 13 or 14, via the methods of Corollary 23. Let $\psi_{q}: K_{0}\left(\operatorname{Var}_{k}\right) \rightarrow \mathbb{Z}$ be the homomorphism

$$
\psi_{q}:[X] \mapsto \# X\left(\mathbb{F}_{q}\right) .
$$

Then $\psi_{q}$ extends to a continuous homomorphism $\widehat{\psi_{q}}: R \rightarrow \mathbb{Z}_{p}$. So it suffices to find an $X$ such that $\widehat{\psi_{q}}\left(\left[\operatorname{Sym}^{n}(X)\right]\right)$ does not converge in $\mathbb{Z}_{p}$.

This happens if the zeta function of $X, \zeta_{X}(t)=\sum_{n=0}^{\infty} \psi_{q}\left(\left[\operatorname{Sym}^{\mathrm{n}}(X)\right]\right) t^{n}$ (which is rational by the Weil conjectures), has a pole at a unit $y \in \mathcal{O}_{\mathbb{C}_{p}}^{\times}$, with $y \neq 1$. But it is easy to construct examples of such $X$ - for example, there are many such Abelian surfaces, by Honda-Tate theory. More simply, the product of two ordinary elliptic curves suffices.

Remark 25. More generally, if $X$ is a smooth projective variety over $k=\mathbb{F}_{q}$, with non-vanishing $h^{0}\left(\Omega_{X}^{2 n}\right)$ for some $n>0$, and the $2 n$-th Newton polygon of the zeta function of $X$ equals its $2 n$-th Hodge polygon (e.g. if $X$ is an ordinary Abelian variety), then we see that False Claims 4 and 5 are indeed false, by the same reasoning as in the previous remark. Note that these examples are of a similar nature to those in Corollary 21. These observations serve as motivation for the results in the next section.

\section{Heuristics for failure of MSSP, And Newton polygons}

In this final section, we will describe a heuristic predicting for which $X$ False Claim 5 (and conditionally MSSP) will fail, in terms of the Hodge numbers of $X$; for $X$ a curve, we will give some evidence for this heuristic.

Definition 26 (Hodge polygon). Let $X / \mathbb{C}$ be a smooth proper variety. Then the $m$-dimensional Hodge polygon of $X$ is the graph of the unique continuous piecewise linear function $\ell_{X, m}$ on $\left[0, \sum_{i+j=m} h^{i, j}\right]$ whose slope on

$$
\left[h^{0, m}+h^{1, m-1}+\cdots+h^{j-1, m-j+1}, h^{0, m}+h^{1, m-1}+\cdots+h^{j, m-j}\right]
$$

is $j$, and which satisfies $\ell_{X, m}(0)=0$.

For example, the 1-dimensional Hodge polygon of a smooth proper curve of genus $g$ has slope 0 on $[0, g]$ and slope 1 on $[g, 2 g]$.

If $X$ is defined over a finite field $\mathbb{F}_{q}$ and admits a lift $X^{0}$ to characteristic zero, there is a well-known relationship between the zeta function $\zeta_{X}(t)$ appearing in the Weil conjectures, and the Hodge polygons of $X^{0}$. Namely, $\zeta_{X}(t)$ is an alternating 
product of polynomials $p_{i}(t)$, where $p_{i}(t)$ is the determinant of $\left(I-\operatorname{Frob}_{q} t\right)$ acting on the $i$-th $\ell$-adic cohomology group of $X_{\overline{\mathbb{F}_{q}}}$ with constant coefficients. Dwork [4], Mazur [14, and Ogus [17] have proven that the Newton polygon of $p_{m}(t)$ (with respect to the valuation at $q$ ) lies above the $m$-dimensional Hodge polygon of $X^{0}$.

We suggest an imprecise analogy with motivic zeta functions, which explains the failure of the convergence of the symmetric powers of $X$. Before expanding on this analogy, we give a precise version for curves (Corollary 33 ). This statement is meant to provide evidence that there is an intimate relationship between the Hodge numbers of a variety and the coefficients of its motivic zeta function, analogous to the work of Dwork, Mazur, and Ogus alluded to above.

Throughout this section, $X$ will be a smooth projective curve with a rational point. We will use the fact that $\operatorname{Pic}^{d}(X)$ admits a stratification by locally closed subvarieties so that the natural map $\operatorname{Sym}^{d}(X) \rightarrow \operatorname{Pic}^{d}(X)$ (sending a divisor to its associated line bundle) is a projective space bundle over each stratum. Furthermore, the fiber over a point $\mathcal{L}$ is naturally identified with $\mathbb{P} \Gamma(X, \mathcal{L})$.

If $f(t) \in K_{0}\left(\operatorname{Var}_{k}\right)[[t]]$ is a power series over $K_{0}\left(\operatorname{Var}_{k}\right)$, we will use $\left[t^{k}\right] f(t)$ to denote the coefficient of $t^{k}$ in $f$.

It is well known ([8, Theorem 1.1.9], [12, Theorem 3.7], [16, Theorem 7.33]) that if $X$ is a smooth projective curve with a rational point,

$$
(1-t)(1-\mathbb{L} t) Z_{X}^{m o t}(t)
$$

is a polynomial in $K_{0}\left(\operatorname{Var}_{k}\right)[t]$ of degree $2 g$; let us compute its coefficients. We have

$$
\begin{aligned}
(1-t)(1-\mathbb{L} t) Z_{X}^{m o t}(t) & =(1-\mathbb{L} t) \sum_{n=0}^{\infty}\left(\left[\operatorname{Sym}^{n}(X)\right]-\left[\operatorname{Sym}^{n-1}(X)\right]\right) t^{n} \\
& =\sum_{n=0}^{\infty}\left(\left[\operatorname{Sym}^{n}(X)\right]-(\mathbb{L}+1)\left[\operatorname{Sym}^{n-1}(X)\right]+\mathbb{L}\left[\operatorname{Sym}^{n-2}(X)\right]\right) t^{n} .
\end{aligned}
$$

Here we take $\left[\operatorname{Sym}^{n}(X)\right]=0$ for $n<0$. In particular, $\left[t^{0}\right](1-t)(1-\mathbb{L} t) Z_{X}^{\text {mot }}(t)=1$. Also,

$$
\begin{gathered}
{\left[\operatorname{Sym}^{2 g}(X)\right]=[\operatorname{Jac}(X)]\left[\mathbb{P}^{g}\right],} \\
{\left[\operatorname{Sym}^{2 g-1}(X)\right]=[\operatorname{Jac}(X)]\left[\mathbb{P}^{g-1}\right],} \\
{\left[\operatorname{Sym}^{2 g-2}(X)\right]=[\operatorname{Jac}(X)]\left[\mathbb{P}^{g-2}\right]+\mathbb{L}^{g-1},}
\end{gathered}
$$

where the last equality follows from the fact that $\omega_{X}$ is the unique degree $2 g-2$ line bundle $\mathcal{L}$ with $h^{0}(X, \mathcal{L})=g$ (from Serre Duality and Riemann-Roch), and all other line bundles $\mathcal{L}^{\prime}$ of degree $2 g-2$ satisfy $h^{0}\left(X, \mathcal{L}^{\prime}\right)=g-1$. So

$$
\begin{aligned}
{\left[t^{2 g}\right](1-t)(1-\mathbb{L} t) Z_{X}^{m o t}(t)=} & {[\operatorname{Jac}(X)]\left[\mathbb{P}^{g}\right]-[\operatorname{Jac}(X)]\left[\mathbb{P}^{g-1}\right] } \\
& \quad+\mathbb{L}\left(-[\operatorname{Jac}(X)]\left[\mathbb{P}^{g-1}\right]+[\operatorname{Jac}(X)]\left[\mathbb{P}^{g-2}\right]+\mathbb{L}^{g-1}\right) \\
= & {[\operatorname{Jac}(X)] \mathbb{L}^{g}+\mathbb{L}\left(-[\operatorname{Jac}(X)] \mathbb{L}^{g-1}+\mathbb{L}^{g-1}\right) } \\
= & \mathbb{L}^{g} .
\end{aligned}
$$

Furthermore, we claim that $\left[t^{g}\right](1-t)(1-\mathbb{L} t) Z_{X}^{m o t}(t)$ is not in $(\mathbb{L})$. For this we need to understand the stable birational geometry of $\operatorname{Sym}^{g-1}(X)$ and $\operatorname{Sym}^{g}(X)$.

Lemma 27. $\operatorname{Sym}^{g-1}(X)$ and $\operatorname{Sym}^{g}(X)$ are not stably birational to one another. 
Proof. $\operatorname{Sym}^{g}(X)$ is birational to $\operatorname{Jac}(X)$, so it suffices to show that $\operatorname{Sym}^{g-1}(X)$ is not stably birational to $\operatorname{Jac}(X)$. Suppose to the contrary that there is a rational, birational map $\operatorname{Sym}^{g-1}(X) \times \mathbb{A}^{n} \rightarrow \operatorname{Jac}(X) \times \mathbb{A}^{n-1}$. Then in particular some open subset $U$ of $\mathbb{A}^{n}$ maps injectively to $\operatorname{Jac}(X) \times \mathbb{A}^{n-1}$; but as $\operatorname{Jac}(X)$ is an Abelian variety, the image of this map under the projection to $\operatorname{Jac}(X)$ is trivial. Thus $U$ is entirely contained in some fiber of the projection, so $U$ maps injectively to $\mathbb{A}^{n-1}$. But $U$ has dimension $n$, so this is impossible.

Remark 28. This argument shows in general that if $X, Y$ are varieties with $\operatorname{dim}(X)>$ $\operatorname{dim}(Y)$, and no rational curves pass through a general point of $X$, then $X$ and $Y$ are not stably birational. In particular, for $C$ a smooth proper curve of genus $g$, and $n \leq g, \operatorname{Sym}^{n-1}(C)$ is not stably birational to $\operatorname{Sym}^{n}(C)$ (no rational curves pass through a general point of $\operatorname{Sym}^{n}(C)$, as it is birational to a subvariety of $\operatorname{Jac}(C)$ ).

Corollary 29. $\left[t^{g}\right](1-t)(1-\mathbb{L} t) Z_{X}^{m o t}(t)$ is not in $(\mathbb{L})$.

Proof. We have that

$$
\left[t^{g}\right](1-t)(1-\mathbb{L} t) Z_{X}^{m o t}(t)=\left[\operatorname{Sym}^{g}(X)\right]-\left[\operatorname{Sym}^{g-1}(X)\right] \bmod \mathbb{L} .
$$

As $\operatorname{Sym}^{g-1}(X), \operatorname{Sym}^{g}(X)$ are smooth and projective, Lemma 27 gives that the image of this difference in $\mathbb{Z}[S B]$ via $s b$ is non-zero, so we have the claim.

Corollary 30 (The Newton polygon of $(1-t)(1-\mathbb{L} t) Z_{X}^{m o t}(t)$ lies below the 1-dimensional Hodge polygon of $X)$. For $x \in K_{0}\left(\operatorname{Var}_{k}\right)$, define $v_{\mathbb{L}}(x) \in \mathbb{Z}_{\geq 0} \cup\{\infty\}$ to be the greatest integer $n$ so that $x \in\left(\mathbb{L}^{n}\right)$. Define the $\mathbb{L}$-adic Newton polygon of a polynomial $p(t)=\sum a_{i} t^{i} \in K_{0}\left(\operatorname{Var}_{k}\right)[t]$ to be the lower convex hull of the set of points $\left(i, v_{\mathbb{L}}\left(a_{i}\right)\right.$ ) (when there is no risk of confusion, we will drop the modifier "LL-adic"). Then the Newton polygon of $(1-t)(1-\mathbb{L} t) Z_{X}^{\text {mot }}(t)$ lies below the 1-dimensional Hodge polygon of $X$.

Proof. Recall that the 1-dimensional Hodge polygon of $X$ consists of the segments $[(0,0),(g, 0)]$ and $[(g, 0),(2 g, g)]$. Thus it suffices to show that $v_{\mathbb{L}}(1)=0$, $v_{\mathbb{L}}\left(\left[t^{g}\right](1-t)(1-\mathbb{L} t) Z_{X}^{m o t}(t)\right)=0$ and $v_{\mathbb{L}}\left(\mathbb{L}^{g}\right)=g$.

That $v_{\mathbb{L}}(1)=0$ is simply the statement that $\mathbb{L}$ is not a unit. To see this, note that it suffices to produce any homomorphism out of $K_{0}\left(\operatorname{Var}_{k}\right)$ sending $\mathbb{L}$ to a nonunit. The homomorphism $K_{0}\left(\operatorname{Var}_{k}\right) \rightarrow \mathbb{Z}[t]$ sending a smooth proper variety to its Poincaré polynomial [13, p. 331] suffices.

Corollary 29 is exactly the statement that $v_{\mathbb{L}}\left(\left[t^{g}\right](1-t)(1-\mathbb{L} t) Z_{X}^{m o t}(t)\right)=0$.

Finally, $v_{\mathbb{L}}\left(\mathbb{L}^{g}\right)$ is greater than or equal to $g$ by definition. But the Poincaré polynomial of $\mathbb{L}^{g}$ is $t^{2 g}$; thus $\mathbb{L}^{g}$ is not in $\left(\mathbb{L}^{g+1}\right.$ ) (which maps to $\left(t^{2 g+2}\right)$ via the Poincaré polynomial), as desired.

In fact, we claim that the Newton polygon of $(1-t)(1-\mathbb{L} t) Z_{X}^{m o t}(t)$ is actually equal to the 1-dimensional Hodge polygon of $X$. To see this, it suffices to show that

$$
v_{\mathbb{L}}\left(\left[t^{n}\right](1-t)(1-\mathbb{L} t) Z_{X}^{m o t}(t)\right) \geq n-g
$$

for $n>g$.

Lemma 31 (The $\mathbb{L}$-adic Newton polygon of $(1-t)(1-\mathbb{L} t) Z_{X}^{m o t}(t)$ lies above the 1-dimensional Hodge polygon of $X)$. For $n>g,\left[t^{n}\right](1-t)(1-\mathbb{L} t) Z_{X}^{m o t}(t) \in\left(\mathbb{L}^{n-g}\right)$. 
Proof. Let $x \in X$ be a rational point. Let $J_{a, b, c} \subset \operatorname{Pic}^{n}(X)$ be the locally closed subset consisting of those line bundles $\mathcal{L}$ with $h^{0}(L)=a, h^{0}(L(-x))=b, h^{0}(L(-2 x))=$ c. Now

$$
\begin{aligned}
{\left[\operatorname{Sym}^{d}(X)\right] } & =\sum_{a, b, c}\left[J_{a, b, c}\right]\left[\mathbb{P}^{a-1}\right], \\
{\left[\operatorname{Sym}^{d-1}(X)\right] } & =\sum_{a, b, c}\left[J_{a, b, c}\right]\left[\mathbb{P}^{b-1}\right], \\
{\left[\operatorname{Sym}^{d-2}(X)\right] } & =\sum_{a, b, c}\left[J_{a, b, c}\right]\left[\mathbb{P}^{c-1}\right],
\end{aligned}
$$

and so

$$
\left[t^{n}\right](1-t)(1-\mathbb{L} t) Z_{X}^{m o t}(t)=\sum_{a, b, c}\left[J_{a, b, c}\right]\left(\left[\mathbb{P}^{a-1}\right]-\left[\mathbb{P}^{b-1}\right]+\mathbb{L}\left(-\left[\mathbb{P}^{b-1}\right]+\left[\mathbb{P}^{c-1}\right]\right)\right) .
$$

By Riemann's inequality,

$$
a \geq n-g+1 ; b \geq n-g ; c \geq n-g-1 ;
$$

furthermore, $0 \leq a-b, b-c \leq 1$. Now if $a=b=c$, the corresponding term in the sum above vanishes. If $a>b$ (that is, $a=b+1$ ) and $b=c$,

$$
\left[\mathbb{P}^{a-1}\right]-\left[\mathbb{P}^{b-1}\right]=\mathbb{L}^{a-1} ;
$$

$a-1=b \geq n-g$, as desired, so the term

$$
\left[J_{a, b, c}\right]\left(\left[\mathbb{P}^{a-1}\right]-\left[\mathbb{P}^{b-1}\right]+\mathbb{L}\left(-\left[\mathbb{P}^{b-1}\right]+\left[\mathbb{P}^{c-1}\right]\right)\right)=\left[J_{a, b, c}\right] \mathbb{L}^{b} \in\left(\mathbb{L}^{n-g}\right) .
$$

An identical argument works for the cases $a=b>c$ or $a>b>c$. Thus $\left[t^{n}\right](1-$ $t)(1-\mathbb{L} t) Z_{X}^{m o t}(t)$ is a sum of terms in $\left(\mathbb{L}^{n-g}\right)$ and so is in $\left(\mathbb{L}^{n-g}\right)$ itself.

Remark 32. This argument works for curves with a rational point in arbitrary characteristic and implies the classical result that the $q$-Newton polygons of the zeta function $\zeta_{X}$ associated to a smooth projective curve $X$ over a finite field $\mathbb{F}_{q}$ lie above its associated Hodge polygons. In particular, the $q$-Newton polygon of $\zeta_{X}(t)=\psi_{q}\left(Z_{X}^{m o t}(t)\right)$ lies above the $\mathbb{L}$-adic Newton polygon of $Z_{X}^{m o t}(t)$ (because $\psi_{q}(\mathbb{L})=q$ ), which lies above the Hodge polygon of $X$.

Corollary 33 (The Newton polygon equals the Hodge polygon of $X$ ). Over algebraically closed fields of characteristic zero, the Newton polygon of $(1-t)(1-\mathbb{L} t) Z_{X}^{m o t}(t)$ consists precisely of the segments $[(0,0),(g, 0)]$ and $[(g, 0)$, $(2 g, g)]$; that is, the $\mathbb{L}$-adic Newton polygon of $(1-t)(1-\mathbb{L} t) Z_{X}^{\text {mot }}(t)$ is the 1 dimensional Hodge polygon of $X$.

Proof. This follows immediately from Corollary 30 and Lemma 31.

Let us now consider the (heuristic) relationship between Newton polygons, MSSP, and False Claims 4 and 5. The important identity here is the following:

$$
\lim _{n \rightarrow \infty}\left[\operatorname{Sym}^{n}(S)\right]=\left.(1-t) Z_{S}^{m o t}(t)\right|_{t=1},
$$

where both sides are evaluated in $R=\widehat{K_{0}\left(\operatorname{Var}_{k}\right)}$, the $\mathbb{L}$-adic completion of $K_{0}\left(\operatorname{Var}_{k}\right)$ (or really any completion of $K_{0}\left(\operatorname{Var}_{k}\right)$ ). 
Imagine for a second that $Z_{S}^{\text {mot }}(t)$ is a rational or meromorphic function. (We will leave these terms purposefully vague.) Then one would expect the identity (4) to hold only if the power series expansion

$$
(1-t) Z_{S}^{m o t}(t)=(1-t) \sum_{n=0}^{\infty}\left[\operatorname{Sym}^{n}(S)\right] t^{n}
$$

is valid near $t=1$. If this identity holds, $Z_{S}^{\text {mot }}(t)$ has no poles $y$ with $v_{\mathbb{L}}(y)<1$. The valuations of such poles are determined by the Newton polygon of the denominator of $Z_{S}^{m o t}(t)$ - there will be a pole of valuation less than 1 if and only if the Newton polygon of the denominator has a segment with slope less than 1 .

Now, by analogy with the Weil conjectures, one expects the denominator of $Z_{S}^{m o t}(t)$ to factor as a product of polynomials relating to the even-degree cohomology of $S$. By analogy to Corollary 33, the Newton polygons of these polynomials should lie below the $2 m$-dimensional Hodge polygons of $S$. These Hodge polygons contain a segment of slope less than 1 (namely, of slope zero) if and only if

$$
h^{0}\left(S, \Omega_{S}^{2 m}\right) \neq 0
$$

for some $m>0$. Suppose now that $h^{0}\left(S, \Omega_{S}^{2 m}\right) \neq 0$ for some $m>0$. Then $(1-t) Z_{S}^{m o t}(t)$ would have a pole of valuation less than 1 , and the power series expansion of $(1-t) Z_{S}^{m o t}(t)$ would not converge at $t=1$.

Question 34. Thus, we ask: does False Claim国 fail for all $S$ with $h^{0}\left(S, \Omega_{S}^{2 m}\right) \neq 0$ ? We have proven this for $S$ of dimension 2 .

While this paper was in preparation, Melanie Wood informed the author 22 of a stronger result than Theorem 19.

Proposition 35. Let $S$ be smooth and projective, with $\operatorname{dim}(S)>1$. Suppose that either $\operatorname{dim}(S)$ is even and $h^{0}\left(S, \omega_{S}^{n}\right)$ is non-zero, or that $h^{0}\left(S, \omega_{S}^{2 n}\right)$ is non-zero for some $n$. Then if $\operatorname{Sym}^{n}(S)$ is stably birational to $\operatorname{Sym}^{m}(S), m=n$.

Applying the arguments of Corollaries 21 and 23, where $S$ is a smooth surface with a non-vanishing plurigenus, gives further examples of failures of False Claim 5 and conditional failures of MSSP. These examples include those given here, as well as e.g. Enriques surfaces and surfaces of general type with vanishing geometric genus. This suggests that our heuristic is incomplete.

Unfortunately, Proposition 35 does not give examples in higher dimensions, as Corollaries 21 and 23] use the existence of a desingularization $\tilde{S}^{n}$ of $\operatorname{Sym}^{n}(S)$ which satisfies $\tilde{S}^{n}=\operatorname{Sym}^{n}(S) \bmod \mathbb{L}$. The existence of such a desingularization in dimension greater than 2 appears to be an open question [12, Question 6.7]. Ulyanov [19. Theorem 2] constructs a compactification of the configuration space of distinct, labeled points in $S$ upon which the symmetric group acts with Abelian stabilizers. He remarks that desingularizing this compactification should be doable via existing methods. So perhaps this question is tractable. Ulyanov's compactification is a modification of the well-known Fulton-MacPherson compactification [6].

\section{ACKNowledgments}

This note owes much to conversations with David Ayala, Rebecca Bellovin, Jeremy Booher, Jonathan Campbell, Brian Conrad, François Greer, Sander Kupers, 
Sam Lichtenstein, Cary Malkiewich, Emmy Murphy, Mircea Mustaţă, Matthew Satriano, Arnav Tripathy, Ravi Vakil, Wim Veys, Kirsten Wickelgren, and Melanie Wood. The author would also like to thank the referee for helpful suggestions.

\section{REFERENCES}

[1] Franziska Bittner, The universal Euler characteristic for varieties of characteristic zero, Compos. Math. 140 (2004), no. 4, 1011-1032, DOI 10.1112/S0010437X03000617. MR2059227 (2005d:14031)

[2] Jan Denef and François Loeser, On some rational generating series occurring in arithmetic geometry, Geometric aspects of Dwork theory. Vol. I, II, Walter de Gruyter GmbH \& Co. KG, Berlin, 2004, pp. 509-526. MR2099079 (2005h:11267)

[3] Albrecht Dold and René Thom, Quasifaserungen und unendliche symmetrische Produkte (German), Ann. of Math. (2) 67 (1958), 239-281. MR0097062 (20 \#3542)

[4] Bernard Dwork, A deformation theory for the zeta function of a hypersurface, Proc. Internat. Congr. Mathematicians (Stockholm, 1962), Inst. Mittag-Leffler, Djursholm, 1963, pp. 247259. MR0175895 (31 \#171)

[5] Barbara Fantechi, Lothar Göttsche, Luc Illusie, Steven L. Kleiman, Nitin Nitsure, and Angelo Vistoli, Fundamental algebraic geometry, Grothendieck's FGA explained. Mathematical Surveys and Monographs, vol. 123, American Mathematical Society, Providence, RI, 2005. MR:2222646 (2007f:14001)

[6] William Fulton and Robert MacPherson, A compactification of configuration spaces, Ann. of Math. (2) 139 (1994), no. 1, 183-225, DOI 10.2307/2946631. MR.1259368 (95j:14002)

[7] Lothar Göttsche, On the motive of the Hilbert scheme of points on a surface, Math. Res. Lett. 8 (2001), no. 5-6, 613-627. MR.1879805 (2002k:14008)

[8] Mikhail Kapranov, The elliptic curve in the S-duality theory and Eisenstein series for KacMoody groups. MSRI Preprint 2000-006, arXiv:math/0001005v2.

[9] Maxim Kontsevich, String Cohomology. Lecture at Orsay, December 7, 1995.

[10] Stéphane Lamy and Julien Sebag, Birational self-maps and piecewise algebraic geometry, J. Math. Sci. Univ. Tokyo 19 (2012), no. 3, 325-357. MR3015002

[11] Michael Larsen and Valery A. Lunts, Motivic measures and stable birational geometry (English, with English and Russian summaries), Mosc. Math. J. 3 (2003), no. 1, 85-95, 259. MR.1996804 (2005a:14026)

[12] Michael Larsen and Valery A. Lunts, Rationality criteria for motivic zeta functions, Compos. Math. 140 (2004), no. 6, 1537-1560. MR2098401(2005k:14045)

[13] Qing Liu and Julien Sebag, The Grothendieck ring of varieties and piecewise isomorphisms, Math. Z. 265 (2010), no. 2, 321-342, DOI 10.1007/s00209-009-0518-7. MR2609314 (2011c:14018)

[14] B. Mazur, Frobenius and the Hodge filtration (estimates), Ann. of Math. (2) 98 (1973), 58-95. MR0321932 (48 \#297)

[15] D. Mumford, Rational equivalence of 0-cycles on surfaces, J. Math. Kyoto Univ. 9 (1968), 195-204. MR0249428 (40 \#2673)

[16] Mircea Mustaţă, Zeta functions in algebraic geometry, http://www.math.lsa.umich.edu/ $\sim$ mmustata/zeta_book.pdf

[17] Arthur Ogus, Frobenius and the Hodge spectral sequence, Adv. Math. 162 (2001), no. 2, 141-172, DOI 10.1006/aima.2001.1975. MR1859245 (2002k:14028)

[18] Jean-Pierre Serre, Grothendieck-Serre correspondence, Amer. Math. Soc. and Soc. Math. France 2004 .

[19] Alexander P. Ulyanov, Polydiagonal compactification of configuration spaces, J. Algebraic Geom. 11 (2002), no. 1, 129-159, DOI 10.1090/S1056-3911-01-00293-4. MR.1865916 (2002j:14004)

[20] Ravi Vakil and Melanie Matchett Wood, Discriminants in the Grothendieck ring, arXiv:1208.3166v1.

[21] Claire Voisin, Hodge theory and complex algebraic geometry. II. Translated from the French by Leila Schneps, Cambridge Studies in Advanced Mathematics, vol. 77, Cambridge University Press, Cambridge, 2003. MR1997577 (2005c:32024b)

[22] Melanie Matchett Wood, personal communication, April 5, 2012. 
Department of Mathematics 380-381M, Stanford University, Stanford, California 94305

E-mail address: dlitt@math.stanford.edu 